\title{
Seamless Hourly Rainfall Ensemble Forecasts
}

\author{
$\underline{\text { S. Cooper }}^{\mathrm{a}}$ and A. Seed ${ }^{\mathrm{a}}$ \\ ${ }^{a}$ Research and Development Branch, Bureau of Meteorology, GPO BOX 1289, Melbourne, Victoria, 3001 \\ Email: S.Cooper@bom.gov.au
}

\begin{abstract}
Typically, Numerical Weather Prediction (NWP) Direct Model Output (DMO) provide deterministic precipitation forecasts. However, these contain no information regarding forecast uncertainty. Additionally, different NWP models have varying spatial and temporal resolutions and forecast period lengths. Many users require a seamless forecast system with a uniform resolution over the entire forecast period. NWP ensemble systems typically produce a limited number of ensemble members, due to computational resources and time constraints, by perturbing initial conditions and/or model physics, providing an insight into the forecast uncertainty. Global NWP ensembles typically contain no more than 50 ensemble members at coarse temporal and spatial resolutions, in regional NWP ensembles that have higher spatial and temporal resolutions at the expense of the number of members, typically less than 25 , and maximum lead time. Limited area ensembles have even higher spatial and temporal resolutions, at the further expense of members and lead time. Some applications, for example hydrological, require high resolution, both spatial and temporal, ensembles with 50-100 members. This is computationally prohibitive for dynamical NWP ensembles.

A large ensemble of rainfall forecasts has been generated by blending two deterministic NWP models with a stochastic model of forecast error, which has the space-time properties of observed rainfall. NWP uncertainties depend on the scale and forecast lead time, especially at long forecast lead times. By blending the models scale by scale, it is possible to recognise the increased skill of the models at short lead times and larger spatial scales. The stochastic model of forecast error is applied at each scale, adding increasingly more variability at small spatial scales and longer forecast lead times, while preserving the space-time structure of rainfall.

The Bureau of Meteorology's Australian Parallel Suite (APS, current version is APS1) Australian Community Climate and Earth-System Simulator (ACCESS) Global model (ACCESS G) $(40 \mathrm{~km} \times \sim 40 \mathrm{~km}, 3$ hourly out to 10 days) and ACCESS-R (Regional) $(\sim 12 \mathrm{~km} \times \sim 12 \mathrm{~km}$, hourly out to 3 days) models are blended over $1000 \mathrm{~km} \times 1000 \mathrm{~km}$ domains. An ensemble of hourly, downscaled precipitation forecasts out to 10 days is produced with a resolution of $2 \mathrm{~km} \times 2 \mathrm{~km}$. A year of these ensemble forecasts (accumulated to daily totals) has been verified against the daily Australian Water Availability Project (AWAP) analyses $\left(0.05^{\circ} \times 0.05^{\circ}\right.$ resolution). These results show the system is able to produce reliable forecasts out to five days over these domains. More recently, hourly (out to 10 days) ACCESS G forecasts (APS1 ACCESS G model outputting hourly fields, running in research mode) and ACCESS R have been used to create hourly forecast ensembles which are being compared to radar observations of rainfall.
\end{abstract}

Keywords: Precipitation, ensemble, stochastic 


\section{INTRODUCTION}

Rainfall is an extremely important weather variable to forecast and also happens to be one of the more difficult and least accurate weather fields predicted by NWP (Ruiz and Saulo, 2012). Rainfall forecasts have implications for decision making in a variety of fields including agriculture, aviation, hydrology and event planning (Berrocal et al., 2008). It is for these reasons that rainfall forecasting has been, and will continue to be, heavily researched.

High spatial and temporal resolution forecasts are required as inputs for both hydrological and flash flood warning systems and these forecasts must have the space-time properties of observed rainfall. Very short term rainfall forecasts, within 2 hours, are predominantly based on the temporal advection of observed radar rainfall fields. Purely advected forecast fields will contain radar rainfall estimation errors, errors in the motion of the field, errors due to the advection, as well as errors due to the growth and decay of the rain fields (Seed, 2003). During the forecast period the rain field will continue to evolve, and the development of a rain field can be influenced by such factors as diurnal cycles, topography and flow regime which will not be accounted for in the advection forecasts (Foresti and Seed, 2014). Once exceeding these time scales, advection nowcasts are unable to successfully predict the future state of the rain fields.

NWP model forecasts are an additional source of information that can be exploited for the purpose of forecasting past the nowcasting period. However, NWP models come with a range of spatial and temporal resolutions, maximum forecast lead times and their own set of forecast uncertainties. Many end users require a seamless forecast that is able to blend the various NWP forecasts into a single forecast with a uniform resolution over the entire forecast period. Some NWP models have high enough spatial resolution that they allow convection permitting schemes to be utilised, while others revert to convection parametrisation. These, and other sources of NWP forecast uncertainty need to be accounted for. Ensemble forecasting is one way of representing the uncertainties and passing this information to users.

Observational studies have shown the evolution of rainfall in space is not independent from its evolution in time. Venugopal et al. (1999) studied tropical convective storms over Darwin with spatial scales of $2-20 \mathrm{~km}$ and temporal scales of $10 \mathrm{~min}$ - several hours. From the storms that were studied, Venugopal et al. (1999) were able to determine that the rate of evolution of the rainfall fields exhibited dynamic scaling, that is the evolution was found to be invariant under space-time transformations of the form $t \sim L^{z}$, where is $t$ time, $L$ is the spatial length and $z$ the is dynamic scaling exponent. The dynamic scaling exhibited by rain fields is quite important as it allows the spatial and temporal organisation to be modelled in a similar fashion to the modelling of turbulence, that is, by employing a multi-fractal approach (Lovejoy and Schertzer, 1995; Schertzer et al., 2001). The fact that rainfall obeys a power law relationship allows downscaling of rainfall fields to take place. For example, it allows small scale space-time variability to be predicted from large scale variability, which is more readily observed and predicted. This is exploited in the Short Term Ensemble Prediction System (STEPS) algorithm of Bowler et al. (2006) through the use of a multiplicative cascade.

\section{Methodology}

By using a multiplicative cascade-based scale decomposition approach, STEPS is able to produce high resolution forecast ensembles. Originally, this system blended an advection forecast from radar observations with a noise model possessing the space-time properties of observed rain fields (Bowler et al., 2004, 2006). This method has since been extended to allow more forecasts to be blended together (Seed et al., 2013). Briefly, the input forecasts are converted to $\mathrm{dBR}$ (using a $10 \log _{10}$ transformation) and decomposed into a hierarchical cascade of features by applying a Gaussian filter to the Fourier Transformations of the fields. By assigning skills to the NWP models at each scale and lead time, it is possible to vary the contribution of the synthetic fields in the forecast blend with scale and lead time. This is very useful as the accuracy of NWP forecasts decrease with forecast lead time and with decreasing spatial scales. By perturbing the NWP cascades with randomly initialized noise fields which have the space-time properties of observed rainfall, an ensemble of precipitation realisations can be generated in a relatively inexpensive manner. These ensembles allow the uncertainties in the forecasts to be communicated to end users (Seed et al., 2013).

Precipitation forecasts from two NWP models, APS1 ACCESS G and ACCESS R, are used as the inputs. ACCESS G has a spatial resolution of $0.375^{\circ}$ latitude and $0.5625^{\circ}$ longitude, approximately $40 \mathrm{~km}$ grid spacing, while ACCESS R has a spatial resolution of $0.11^{\circ}$ latitude and $0.11^{\circ}$ longitude, approximately $12 \mathrm{~km}$ grid spacing. ACCESS G forecasts out to 240 hours with 3 hourly timesteps while ACCESS R produces hourly forecasts out to 75 hours (NMOC, 2010). These forecasts are reprojected onto a $1000 \mathrm{~km} \times 1000 \mathrm{~km}$ grid with 
a spatial resolution of $2 \mathrm{~km} \times 2 \mathrm{~km}$ using an Albers Equal Area projection (Snyder, 1987). The Albers Equal Area projection is a projection is an area preserving projection making it suitable for use with area sensitive applications such as rainfall accumulations. ACCESS G forecasts are then temporally interpolated to hourly forecasts using an optical flow algorithm which tracks and extrapolates the apparent motion of the rainfall field (Bowler et al., 2004). Each model is assigned skills that vary with forecast lead time and cascade level, altering the amount of stochastic noise that is injected into the blend of the NWP models. Hourly precipitation ensembles are produced and are referred to as Seamless forecasts.


Figure 1. The NEAus (left) and SEAus (right) domains.

By comparing a year's worth of NWP forecasts the Australian Water Availability Project (AWAP) rainfall analysis, multiplicative bias correction fields have been calculated. The AWAP analysis is a dataset of daily rainfall produced on a $0.05^{\circ} \times 0.05^{\circ}(\sim 5 \mathrm{~km} \times \sim 5 \mathrm{~km})$ grid by interpolating rain gauge observations. An anomaly based approach is used, where the rainfall field is decomposed into a long term average and an associated anomaly, based on the rain gauges. This approach leads to a smoother analysis than the true rain field. Full details of the approach are described by Jones et al. (2009). These analyses are 24 hour rainfall accumulations and monthly accumulations over the entire Australian domain. The maximum temporal resolution from AWAP is daily, whereas the Seamless forecasts are hourly. Furthermore, its resolution is still coarser than the output resolution of the Seamless forecasts. To initially test the accuracy of the Seamless forecasts, it was decided that accumulating Seamless forecasts to daily totals and comparing them to the AWAP analysis was appropriate as this is the only gridded analysis with coverage of the Australian continent.

The bias correction was determined by comparing the NWP forecast to the AWAP analysis for a 12 month period, April 12012 - March 2013, using grid points at which the forecast and analysis both recorded rainfall more than $2 \mathrm{~mm}$ per day. To achieve this, the AWAP analysis was projected onto the $2 \mathrm{~km} \times 2 \mathrm{~km}$ output (forecast ensemble) grid. The ratio of NWP to AWAP analysis was calculated to determine the multiplicative field. As there were few rain events at some pixels, a lower threshold for the number of events required before calculating the bias field was set to 10 . Additionally, if the ratio calculated was less than $1 / 3$ or greater than 3 , the ratio was ignored so as the restrain the amount of variance in the bias field and to ensure that forecast misses were not captured in the bias field, for example, if a NWP forecast was spatially incorrect and the edge of the forecast rain event coincided with the observed event.

A year's worth of ensembles, from April 12012 - March 31 2013, were generated over two $1000 \mathrm{~km} \times$ $1000 \mathrm{~km}$ domains labelled NEAus and SEAus, see Figure 1. These 50 member ensembles of hourly rainfall forecasts out to 10 days with a $2 \mathrm{~km} \times 2 \mathrm{~km}$ resolution were verified against the AWAP analysis. The same period for the calculation of the bias correction field and for the subsequent forecast verification was used. Although this is not ideal, it was necessary as the then current versions of the ACCESS G and ACCESS R models were used at the time. Unfortunately, there was not a longer history which would have allowed a 12 month bias field calculation and an independent 12 month forecast verification at the time. Model upgrades have since taken place. 


\section{VERIFICATION}

\subsection{Daily}

The hourly ensemble forecasts were accumulated to 24 hour totals that matched the AWAP analysis, which is a daily rainfall accumulation from 9 am - 9 am local time. For the comparison the AWAP analysis was re-projected onto the same grid as the forecasts and a conditional verification approach was used. Points were only considered if either an ensemble member or the AWAP analysis had a rainfall event, an accumulation of more than $2 \mathrm{~mm}$, during the day. That is, if all the ensembles forecast rainfall less than $2 \mathrm{~mm}$ and less than 2 $\mathrm{mm}$ was observed at that point, the data point was not considered.


Figure 2. The reliability curves for the 24 hour forecast period ending at 45 hour (left) and 69 hour (right) forecast lead time for the NEAus domain. The 5, 10 and $20 \mathrm{~mm}$ thresholds are shown. The dashed diagonal line indicates the line of perfect reliability and the horizontal dashed coloured lines indicate the observed frequency of the respective thresholds over the verification period. The bar plots in the background show the number of forecast (scale on the right hand side) that fell into the forecast probability bins for each threshold.

Figure 2 shows the reliability curves for the 5, 10 and $20 \mathrm{~mm}$ thresholds for the 12 month period over the NEAus domain for the 24 hour forecast period ending at the 45 hour forecast lead time, left, and the 69 hour forecast lead time, right. The bar plots in the background indicate the number of forecasts (scale on the righthand side of the image) that fell within the probability bins for the respective thresholds. The dashed diagonal line indicates perfect reliability, i.e. each time a given probability was forecasts the observed relative frequency was the same. The dashed horizontal lines indicate the observed frequency of the respective thresholds over the verification period. These plots show that the forecasts fields, in general, are reliable as compared to the AWAP analysis as the lines for each threshold are close to the dashed diagonal line. The reliability curve on the left indicates the Seamless forecasts are under forecasting the probabilities at the low probabilities and a slight over forecasting at the medium to high probabilities. The curve on the right shows that the forecasts probabilities are slightly lower than those observed for almost all probability bins. This is the case out to day 5 , after which the reliability of the ensemble forecasts decreases.

Along with being reliable, it is important that the forecast ensemble is able to communicate the forecast uncertainty to end users. That is, the spread in the ensemble should closely match the forecast error. Spreadreliability plots show how the system is performing by plotting the Root Mean Square (RMS) error against the RMS ensemble spread. Figure 3 show these plots for the two forecast domains for six 24 hour forecast accumulations. As can be seen in the NEAus domain plot (left), the ensemble is consistently underspread for much of the forecast period. However, it is possible to increase the spread in the forecasts by decreasing the assigned skills of the NWP models in the forecast blend, which will increase the stochastic component of the blend. Over the SEAus domain, Figure 3 (right), the spread is much more closely matched to the error. Interestingly, it is the earliest 24 hour accumulation that was underspread, indicating the weights of the NWP models were too high in the early forecast lead times of the Seamless forecasts. 

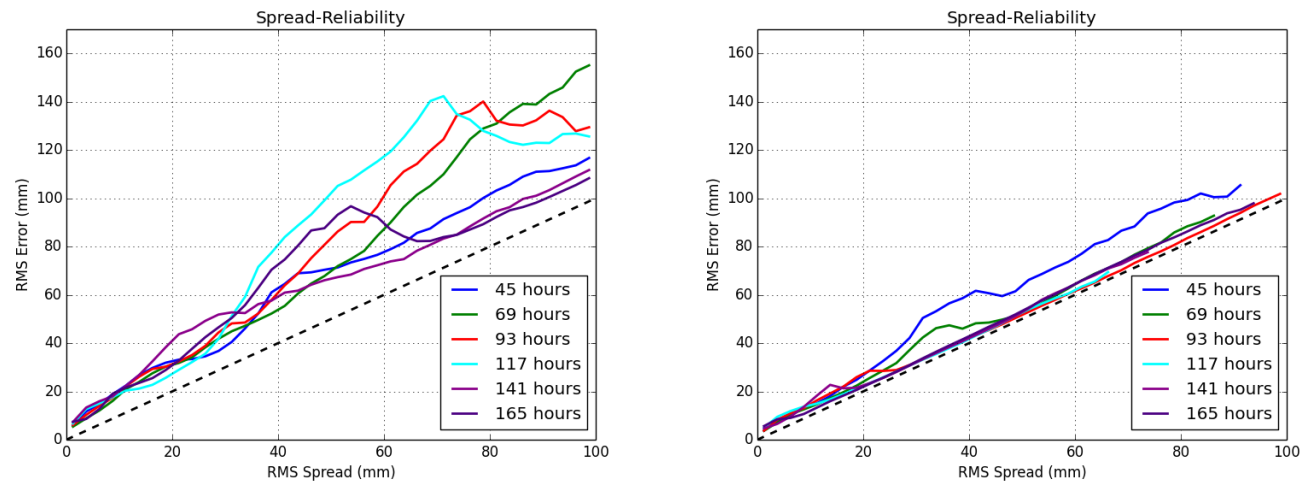

Figure 3. The spread-reliability over the NEAus (left) and SEAus (right) domains. The legend references the final lead time in the 24 hour accumulation. That is, the 45 hours line is from the $21-45$ hour forecasts.

\subsection{Hourly}

The nature of the AWAP analysis means the fields that it produces are smoother than reality, and it can also lose some of the highest rain rates. Radar observations of rainfall are available over some parts of Australia and are another source of data that can be compared to the forecasts. However, as with the AWAP analysis, radar observations have their own limitations that need to be taken into account. These include beam blocking, Z-R calibrations, beam widening at long range, ground clutter and anomalous propagation. However, the radar observations give much higher spatial and temporal resolutions, albeit over a limited spatial domain.

The radar observations produce a rainfall analysis based on the reflectivity. These rainfall observations are then bias corrected against a network of ground-based rain gauges. A mosaic of three radars over Sydney produces hourly accumulations of rainfall over a relatively large domain (as compared to single radar domains). Hourly ACCESS G forecast out to 240 hours are used from the APS1 research mode ACCESS G, along with ACCESS R forecasts, to produce over the Sydney domain to allow a comparison with these observations. These Seamless forecasts are created using the raw precipitation forecasts from the models, that is, no bias correction is applied.

Figure 4 gives an example of a Seamless ensemble member forecast, at the 19 hour forecast lead time, for a rain event over the Sydney domain on April 20, 2015. The ACCESS G (top left) and ACCESS R (top right) forecasts that were used as inputs are shown, along with the radar observation (bottom right). The dark circle in all plots indicating the position of the radar.

The ACCESS $G$ forecast has a large band of rain, from the top left to bottom right, throughout the radar domain, with a similar band of rainfall shown in the radar observation itself. It should be noted that the radar observations are tuned for high impact weather, and as such perform better at higher rain rates, with low rain rates being underestimated. The coarse resolution of the ACCESS G model is evidenced by the grid cell sizes in the forecast. The grid cells are also evident ACCESS R forecast, however these are significantly reduced compared to ACCESS G. ACCESS R also has a similar band of rainfall predicted over the domain. Additionally, it has a single grid cell forecasting rainfall above $50 \mathrm{~mm}$ for the hour, north east of the radar's position. The higher resolution of this model allows for more structure in the rain field, compared to the ACCESS $G$ forecast. The Seamless forecast (bottom left) exhibits the band of rainfall from the two input NWP forecasts. The much higher resolution of the forecast is evident by the structures that are modelled in the rain field. Areas of heavier precipitation are located within areas of lighter precipitation, as is observed in real rain fields. This member has forecast a stronger band of precipitation north east of the radar's position, with a similar band of stronger precipitation observed by the radar, albeit slightly south west of the Seamless forecast. The rain fraction, the ratio of the number of raining pixels to the number of non-raining pixels, of the Seamless forecast is higher than the radar, however as previously noted, the radar does underestimate light rain rates. The raining fraction of the Seamless forecast is heavily influenced by the input NWP model, which both have raining fractions that are higher than those observed by the radar.

The Seamless forecasts rain fields look more realistic than the raw NWP forecasts and have the added advantage of being an ensemble forecast. The main structures of the input NWP models can be recognised within 

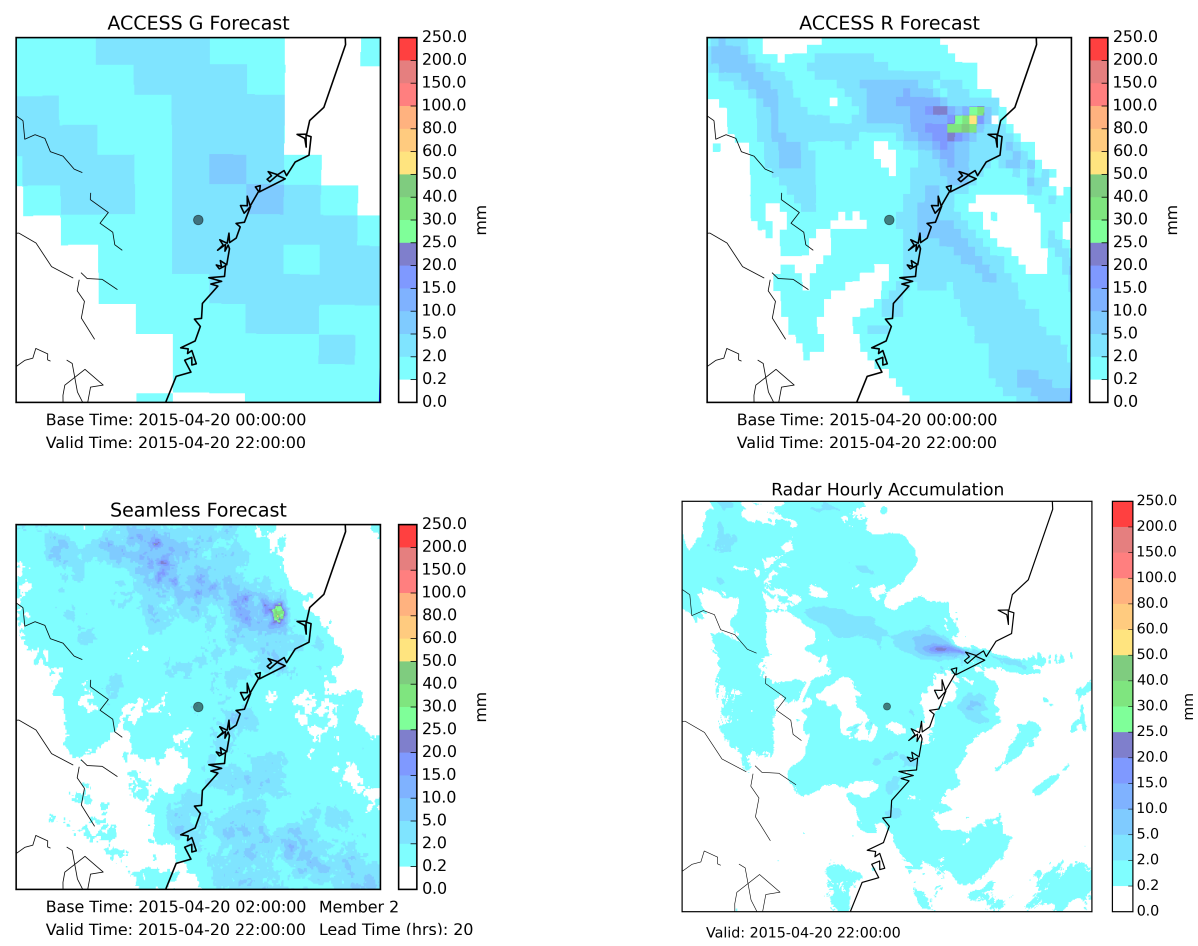

Figure 4. Input NWP forecast from ACCESS G (top left), ACCESS R (top right) along with an example ensemble member forecast (bottom left) and the hourly radar observation (bottom right). The location of the radar is indicated by the dark circle in each image.

the Seamless forecast as the skills of the NWP models at this forecasts lead time are still relatively high. The Seamless system relies on the input NWP models for its forecasts, so it is important that the NWP forecasts have some skill. The NWP forecasts are deterministic and may not properly capture each rain event. Therefore, the idea is to use a range of input NWP models to further capture uncertainties in the forecasts.

\section{CONCLUSIONS}

Many end users require forecasts that have the same temporal and spatial resolution throughout the entire forecast length and also require information about the forecast uncertainty. For ensembles to be of use, they must be reliable and the ensemble spread should encompass the forecast error. Seamless rainfall forecasts blend two NWP models with a stochastic model of forecast error to produce an ensemble of realisations possessing the space-time properties of observed rainfall. These forecasts have the same spatial and temporal resolution throughout the entire forecast period. A year's worth of ensemble forecasts, accumulated to 24 hour totals, were compared to AWAP analyses and showed the forecasts are reliable out to 5 days. The spread in the ensemble was compared to the RMSE and this showed the ensembles were underspread in the NEAus domain, but performed much better over the SEAus domain. As NWP models do not forecast rain as accurately at tropical latitudes, the spread in the NEAus domain can be increased by lowering the skills of the NWP models in the blend.

The ensemble forecasts look far more realistic when compared to radar observations as they have the spacetime properties of observed rainfall and higher resolution than the input NWP fields. Furthermore, it is a relatively inexpensive method of generating ensembles, allowing forecast uncertainties to be communicated to end users. In the future, extra NWP models will be blended to increase the spread of the ensemble and further capture forecast uncertainties.

\section{ACKNOWLEDGEMENT}

The authors would like to thank Mark Curtis and Philip Riley for all their assistance throughout the preparation of this paper. 


\section{REFERENCES}

Berrocal, V. J., A. E. Raftery, and T. Gneiting (2008). Probabilistic quantitative precipitation field forecasting using a two-stage spatial model. The Annals of Applied Statistics 2(4), 1170-1193.

Bowler, N. E., C. E. Pierce, and A. Seed (2004). Development of a precipitation nowcasting algorithm based upon optical flow techniques. J. Hydrol. 288, 74-91.

Bowler, N. E., C. E. Pierce, and A. W. Seed (2006). STEPS: A probabilistic precipitation forecasting scheme which merges an extrapolation nowcast with downscaled NWP. Q. J. R. Meterol. Soc. 132, 2127-2155.

Foresti, L. and A. Seed (2014). On the spatial distribution of rainfall nowcasting errors due to orographic forcing. Meteorol. Appl..

Jones, D., W. Wang, and R. Fawcett (2009). High-quality spatial climate data-sets for australia. Australian Meteorological and Oceanographic Journal 58, 233-248.

Lovejoy, S. and D. Schertzer (1995). Multifractals and rain. In Z. W. Kundzewicz (Ed.), New Uncertainty Concepts in Hydrology and Water Resources, pp. 61-103. Cambridge University Press.

NMOC (2010). Operational implementation of the ACCESS Numerical Weather Prediction systems. NMOC Operations Bulletin 83, Bureau of Meteorology. (http://www.bom.gov.au/australia/charts/bulletins/apob83.pdf).

Ruiz, J. J. and C. Saulo (2012). How sensitive are probabilistic precipitation forecasts to the choice of calibration algorithms and the ensemble generation method? Part I: sensitivity to calibration methods. Meteorol. Appl. 19, 302-313.

Schertzer, D., S. Lovejoy, and P. Hubert (2001). An introduction to stochastic multifractal fields. In Proceedings of the ISFMA Symposium on Environmental Science and Engineering with related Mathematical Problems, Series in Contemporary Applied Mathematics. High Education Press.

Seed, A. (2003). A dynamic and spatial scaling approach to advection forecasting. J. Appl. Meteor. 42, $381-388$.

Seed, A. W., C. E. Pierce, and K. Norman (2013). Formulation and evaluation of a scale decomposition-based stochastic precipitation nowcast scheme. Water Resour. Res. 49.

Snyder, J. P. (1987). Map projections: A working manual. Professional paper 1395, U. S. Geological Survey.

Venugopal, V. E., E. Foufoula-Georgiou, and V. Sapozhinkov (1999). Evidence of dynamic scaling in spacetime rainfall. J. Geophys. Res. 104(D24), 31599-31610. 